\title{
Erratum to: Characteristics of Symmetric Surface Plasmon Polariton Mode in Glass-Metal-Glass Waveguide
}

\author{
Md. Ghulam Saber • Rakibul Hasan Sagor
}

Published online: 18 October 2013

(C) Springer Science+Business Media New York 2013

\section{Erratum to: Plasmonics}

\section{DOI 10.1007/s11468-013-9579-x}

The original version of this article unfortunately contained a mistake. In the 'material models' section, equation (2) should be replaced by the following equation.

$$
\varepsilon_{r}(\omega)=1-\frac{f_{o} \omega_{p}^{2}}{\omega^{2}-j \Gamma \omega}+\sum_{i=1}^{5} \frac{f_{i} \omega_{p}^{2}}{\omega_{o i}^{2}+j \Gamma_{i} \omega-\omega^{2}}
$$

